\title{
The Peptide Alkaloid Anachelin: NMR Spectroscopic Evidence for $\beta$-Turn Formation in Aqueous Solution
}

\author{
Karl Gademann* and Herbert Budzikiewicz ${ }^{\mathrm{a}}$
}

\begin{abstract}
Anachelin, a complex secondary metabolite isolated from Anabaena cylindrica, possesses an unusual structure combining polyketide, peptide and alkaloid building blocks. This natural product was postulated to act as siderophore with catecholate and salicylate groups for putative iron binding with the three-dimensional solution structure of anachelin remaining unknown. In this preliminary communication, the conformation of anachelin in aqueous solution is investigated. ROESY experiments revealed several interresidual NOEs characteristic of a compact, folded structure. A $\beta$-turn type structure of the central tripeptide sequence L-Thr-D-Ser-L-Ser structure involving $\mathrm{D}-$ Ser at position $(i+1)$ and L-Ser at position $(i+2)$ would be in agreement with the observed experimental data. In particular, this conformation of the free ligand would align the putative metal binding groups (structural preorganization) and thus lower the free energy for Fe binding. As it is highly unusual to see such a small tripeptide fragment folding into a compact structure, it appears reasonable to assume that both the cationic tetrahydroquinolinium ring as well as the trihydroxylated $\varepsilon$-amino acid contribute to the stabilization of the folded structure.
\end{abstract}

Keywords: Anachelin · Conformational analyses · Cyanobacteria · Natural product · NMR spectroscopy $\beta \beta$-Turn

Over two billion years ago, cyanobacteria probably introduced oxygenic photosynthesis on earth [1]. This had the detrimental side effect that the soluble Fe(II) salts were oxidized to the corresponding $\mathrm{Fe}(\mathrm{III})$ species of which the dominant iron oxide hydrates are poorly soluble in aqueous solution at physiological $\mathrm{pH}[2]$. Thus, iron acquisition became a major challenge for every organism. To accommodate this problem, life has evolved sophisticated strategies for iron uptake, transport, and storage. Microorganisms use low molecular weight ligands (so called siderophores) released in their extracellular surroundings for iron acquisition [2].

Although cyanobacteria have been present on earth for over two billion years and constitute one of its most successful life forms, it is surprising to see that the molecular mechanism of cyanobacterial iron acquisition is largely unknown [3]. Until recently, only simple compounds such as hydroxamic acids and shizokinen were thought to be involved in cyanobacterial Fe acquisition [3].
This changed three years ago, however, when a complex secondary metabolite was isolated from Anabaena cylindrica and postulated to act as a siderophore. Budzikiewicz and coworkers isolated anachelin-1 (1) together with its hydrolysis product, anachelin H (3) [4]. Later, a Japanese group independ-

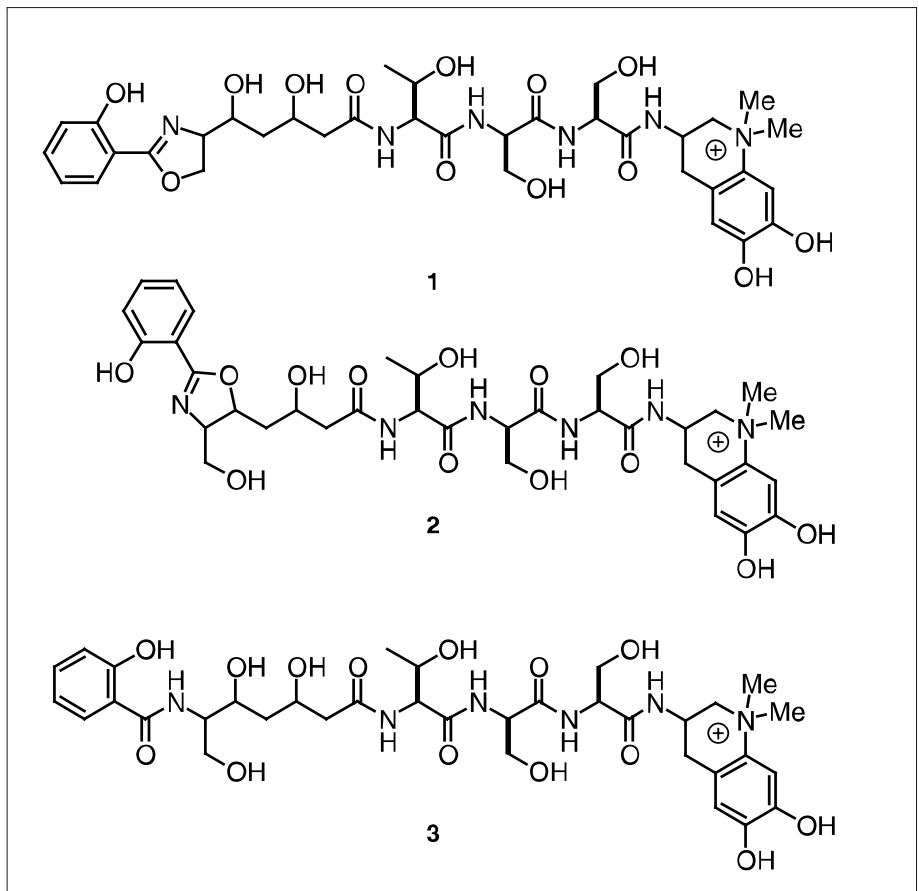


ently reported the structure elucidation of anachelin-1 (1) and anachelin-2 (2), which are constitutional isomers [5]. While they did not isolate anachelin $\mathrm{H} \mathrm{(3)}$, two related esters of salicylic acid with OH-5 and OH-7 of Gla, constitutional isomers of $\mathbf{3}$, were also characterized. Up to now, it has not been determined which of these compounds is biologically active as a bacterial growth factor. Moreover, it is not clear whether these compounds $\mathbf{1 - 3}$ are actually congeners or were formed in the course of the isolation procedures.

The architecture of anachelin is characterized by a fascinating blend of different modular substructures originating from alkaloid, polyketide and peptide biosynthetic pathways. As the mode of action remains elusive, there is little known about the function of each of these modules. As catecholate and salicylate groups are often encountered in siderophores, it is reasonable to assume that these participate in iron complexation of anachelin. The structural tasks, however, of the trihydroxylated amino acid as well as of the tripeptide segment are unclear. For the latter fragment L-Thr-D-Ser-LSer several possible functions or combinations thereof are feasible: (i) it could simply serve as a 'dummy' spacer linking the two putative binding sites, (ii) the side-chains could be involved in Fe complexation, (iii) the amino acids could be part of the binding and recognition by the host cell in the course of Fe-complex uptake, (iv) the tripeptide or the 1,3-diol could serve as cleavage point for ligand breakdown in the course of intracellular Fe release, (v) or this segment could induce a favored conformation in order

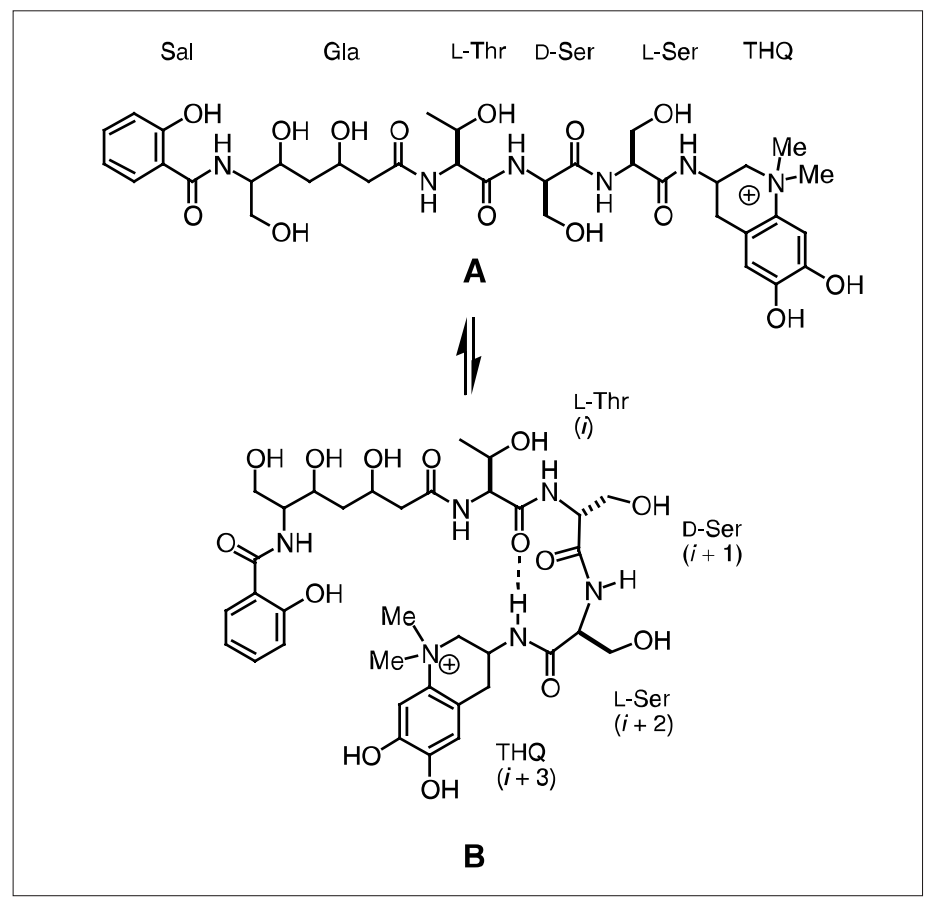

Scheme. Two possible conformational types for anachelin: An extended conformation (A) or a compact, folded structure, e.g. a $\beta$-turn (B). Abbreviations: $\mathrm{THQ}=3$-amino-7,8-dihydroxy-3,3-dimethyl-tetrahydroquinolinium; Gla = 6-amino-3,5,7-trihydroxyheptanoic acid; Sal = salicylic acid.

to facilitate metal binding. Whereas the putative iron binding groups are remote in a linear conformation (A in the Scheme), a folded and more compact conformation $\mathbf{B}$ would favor Fe-binding due to entropic reasons.

The research program of one of our groups (K.G.) is directed towards elucidat- ing the molecular mechanism of cyanobacterial iron acquisition, and so we became interested in the solution structure of anachelin. In this preliminary communication, we will discuss NMR spectroscopic evidence for a folded and compact secondary structure of the peptide alkaloid anachelin in aqueous solution.
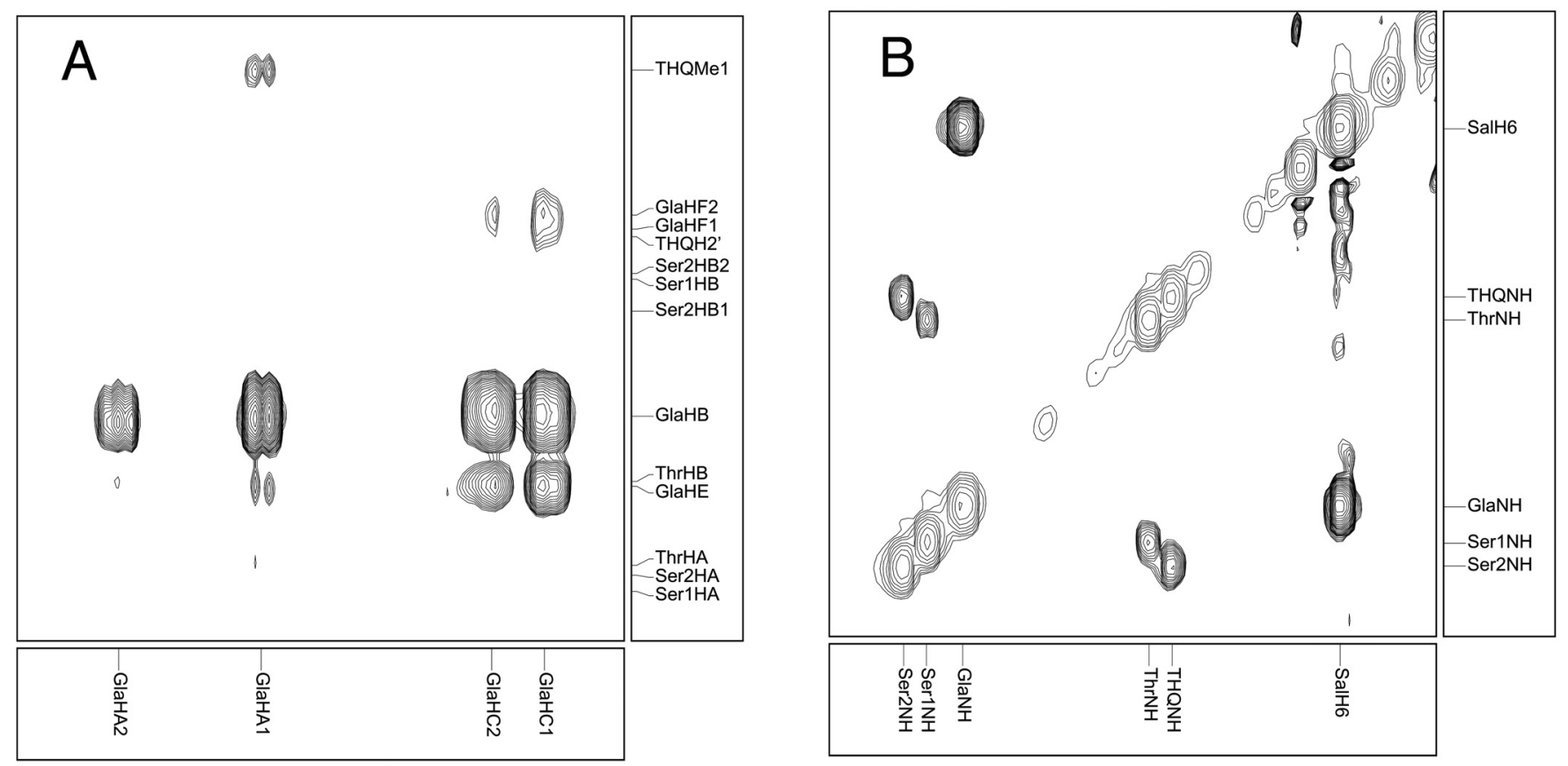

Fig. 1. Representative ROESY data of 3 in $\mathrm{H}_{2} \mathrm{O} / \mathrm{D}_{2} \mathrm{O}$ 9:1, $298 \mathrm{~K}$. A: Cross-strand NOE between the THQ methyl group and the $\mathrm{H}-\mathrm{C}(\alpha)$ of $\mathrm{Gla}$. $\mathrm{B}$ : NOEs supporting the turn structure, such as an NOE from NH of Thr to NH of Ser1, as well as from NH of THQ to the NH of Ser2. For abbreviations, see Scheme. 


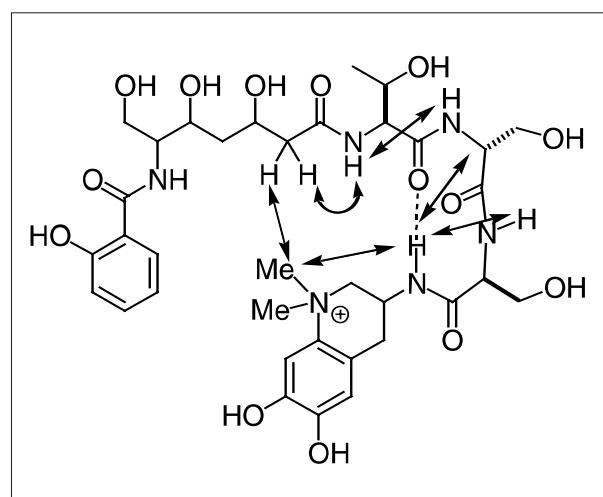

Fig. 2. Schematic presentation of key NOEs observed for anachelin $\mathrm{H}$ (3) suggesting a compact, folded conformation.

Fig. 1 shows representative ROESY spectra of anachelin $\mathrm{H}$ (3) recorded in $\mathrm{H}_{2} \mathrm{O} / \mathrm{D}_{2} \mathrm{O} 9: 1$ using a $500 \mathrm{MHz}$ Bruker spectrometer at $298 \mathrm{~K}$. Several interesting long range cross peaks, shown in the schematic diagram in Fig. 2, were observed: (i) a weak NOE from the methyl group of the tetrahydroquinolinium (THQ) system to the $\mathrm{H}-\mathrm{C}(\alpha)$ of the $\varepsilon$-amino acid derivative (Gla) (Fig. 1A); (ii) a medium NOE from NH of THQ to the NH of Ser2 (Fig. 1B) and (iii) a medium NOE from $\mathrm{NH}$ of Thr to the NH of Ser1 (Fig. 1B). It is interesting to note that the corresponding NOEs between the NH of Ser1 and Ser2 could not be detected. Last, several cross peaks from the NH of THQ to $\mathrm{H}-\mathrm{C}(\alpha)$ and $\mathrm{H}-\mathrm{C}(\beta)$ of both serine residues were also observed.

These NOEs are clear evidence for the presence of a compact and folded conformation of anachelin $\mathrm{H} \mathrm{(3)}$ in aqueous solution. Although NOEs can be a result of conformational averaging [6], a $\beta$-turn confor- mation involving D-Ser at position $(i+1)$ and L-Ser at position $(i+2)$ could explain the presence of these cross peaks (B in the Scheme). In fact, the use of D-amino acids in order to promote $\beta$-turn formation is a well-used practice in order to favor a turn conformation [7]. However, the determination of the exact conformation of anachelin remains difficult at the moment. Computational studies combined with NMR data such as NOE restrained molecular dynamics should allow for the differentiation between several structural possibilities, e.g. type I and II $\beta$-turns and corresponding reverse turns.

It is spectacular to see that a small tripeptide containing one $\mathrm{D}$-amino acid is enough to induce a compact and folded structure. Although the driving force for folding is unknown, it is reasonable to speculate that the presence of the unique cationic tetrahydroquinolinium fragment THQ as well as the trihydroxylated amino acid Gla also contribute to the free energy of the folded structure [8]. Again, additional NMR measurements combined with computational studies could investigate the role of these fragments on the globular folded structure. These experiments as well as the structure determination are currently underway in our laboratory and will be reported in due course.

\section{Acknowledgements}

K.G. is indebted to Prof. Dr. Erick M. Carreira for providing generous financial support. A research grant to K.G. from the Schweizerischer Nationalfonds zur Förderung der Wissenschaftlichen Forschung is gratefully acknowledged.

Received: January 19, 2004
[1] R.E. Summons, L.L. Jahnke, J.M. Hope, G.A. Logan, Nature 1999, 400, 554-557; R.E. Blankenship, H. Hartman, TIBS 1998, 23, 94-97 and references cited therein.

[2] R. Crichton, 'Inorganic Biochemistry of Iron Metabolism: From Molecular Mechanism to Clinical Consequences', 2nd. Ed., John Wiley \& Sons, New York, 2001.

[3] N.M. Price, F.M.M. Morel, Metal Ions Biol. Syst. 1998, 35, 1-36, S.W. Wilhelm, D.P. Maxwell, C.G. Trick, Limnol. Oceanogr. 1996, 41, 89-97; S.W. Wilhelm, K. MacAuley, C.G. Trick, Limnol. Oceanogr. 1998, 43, 992-997.

[4] H. Beiderbeck, K. Taraz, H. Budzikiewicz, A.E. Walsby, Z. Naturforsch. 2000, 55c, 681-687, H. Beiderbeck, Dissertation, Universität zu Köln, Dr. Dirk Blunk Verlag, 2000.

[5] Y. Itou, S. Okada, M. Murakami, Tetrahedron 2001, 57, 9093-9099.

[6] For a detailed overview, see: 'Two-Dimensional NMR spectroscopy - Applications for Chemists and Biochemists', Eds. W.R. Croasmun, R.M.K. Carlson, VCH, New York, 1994, Chap. 5 and 6.

[7] For a review articles, see: G.D. Rose, L.M. Gierasch, J.A. Smith, Adv. Protein Chem. 1985, 37, 1-109; J. Venkatraman, S.C. Shankaramma, P. Balaram, Chem. Rev. 2001, 101, 3131-3152.

[8] It is interesting to note that a tetrapeptide (e.g. Asn-Pro-Asp-Met), obviously lacking flanking residues, did not show any sign of $\beta$-turn formation, see: X. Liu, P.G. Scott, A. Otter, G. Kotovych, Biopolymers 1992, 32, 119. 